\title{
Factors Predicting Ethiopian Anesthetists' Intention to Leave Their Job
}

\author{
Adrienne Kols ${ }^{1}$ - Sharon Kibwana ${ }^{1,2} \cdot$ Yohannes Molla $^{2}$. \\ Firew Ayalew ${ }^{2} \cdot$ Mihereteab Teshome $^{2} \cdot$ Jos van Roosmalen $^{3}$. \\ Jelle Stekelenburg ${ }^{4,5}$
}

Published online: 6 November 2017

(C) The Author(s) 2017. This article is an open access publication

\begin{abstract}
Background Ethiopia has rapidly expanded training programs for associate clinician anesthetists in order to address shortages of anesthesia providers. However, retaining them in the public health sector has proven challenging. This study aimed to determine anesthetists' intentions to leave their jobs and identify factors that predict turnover intentions.

Methods A nationally representative, cross-sectional survey of 251 anesthetists working in public-sector hospitals in Ethiopia was conducted in 2014. Respondents were asked whether they planned to leave the job in the next year and what factors they considered important when making decisions to quit. Bivariate and multivariable logistic regressions were conducted to investigate 16 potential predictors of turnover intentions, including personal and facility characteristics as well as decision-making factors.

Results Almost half $(n=120 ; 47.8 \%)$ of anesthetists planned to leave their jobs in the next year, and turnover intentions peaked among those with 2-5 years of experience. Turnover intentions were not associated with the compulsory service obligation. Anesthetists rated salary and opportunities for professional development as the most important factors in decisions to quit. Five predictors of turnover intentions were significant in the multivariable model: younger age, working at a district rather than regional or referral hospital, the perceived importance of living conditions, opportunities for professional development, and conditions at the workplace.

Conclusions Human resources strategies focused on improving living conditions for anesthetists and expanding professional development opportunities may increase retention. Special attention should be focused on younger anesthetists and those posted at district hospitals.
\end{abstract}

Electronic supplementary material The online version of this article (doi:10.1007/s00268-017-4318-7) contains supplementary material, which is available to authorized users.

Sharon Kibwana

Sharon.kibwana@jhpiego.org

Jhpiego, 1615 Thames St \# 200, Baltimore, MD 21231, USA

2 Jhpiego Ethiopia, Kirkos Subcity, Kebele 02/03, House 693, Wollo Sefer, Addis Ababa, Ethiopia

3 Athena Institute, VU University, Amsterdam, The Netherlands
4 Department of Obstetrics and Gynecology, Leeuwarden Medical Centre, Leeuwarden, The Netherlands

5 Department of Health Sciences, Global Health, University Medical Centre Groningen, University of Groningen, Groningen, The Netherlands 


\section{Introduction}

A shortage of anesthesia providers contributes to a crisis in access to surgical services in low- and middle-income countries (LMICs). The Lancet Commission on Global Surgery has estimated that $94 \%$ of the population in LMICs lacks access to safe, affordable anesthesia and surgical care [1]. Surgically treatable conditions, including in the field of obstetrics, however, make up an increasing proportion of the burden of disease in these settings [2]. To increase availability of anesthesia services, LMICs have shifted tasks from anesthesiologists to associate clinician cadres, such as anesthetists [3], and these providers now deliver most anesthesia services worldwide [4, 5]. But high turnover-driven by poor working conditions, limited remuneration, and low morale among other factors-has undermined efforts to expand the number of anesthesia providers in some LMICs [1, 6, 7].

Designing strategies to reduce turnover among anesthesia providers requires a good understanding of the local context because education, supervision, support, and working conditions vary widely [1, 4, 6-10]. Information about this essential cadre, however, remains limited [5]. This paper examines the situation in Ethiopia, where a shortage of anesthesia providers limits surgical and obstetric services [11]. Although estimates vary, there are certainly very few practicing physician anesthesiologists in Ethiopia and most hospitals rely exclusively on associate clinician anesthetists [11]. The Federal Ministry of Health (FMOH) has set explicit goals to increase the number of anesthetists by more than fivefold by 2025 [12]. To accomplish this, the FMOH expanded the number of preservice education programs for anesthetists from two in 2006 to 26 in 2015 and introduced accelerated anesthetist training options for experienced nurses [13]. It has also implemented multiple interventions to strengthen the quality of anesthetist education.

Retaining newly trained anesthetists in the public sector has proven challenging [14, 15] despite a compulsory service obligation requiring anesthetists who graduate from public training institutions to serve 1-4 years at a public hospital, depending on the length of training, region, and sponsoring organization. This paper analyzes data on Ethiopian anesthetists' turnover intentions, that is, whether they intend to leave their current job in the next year. This is widely accepted as the best available predictor of actual turnover [16]. We explore three questions: What proportion of anesthetists at public-sector hospitals in Ethiopia intend to leave their jobs in the next one year? What factors do they consider important when making decisions to remain in or leave their jobs? What factors predict anesthetists' intentions to leave their job?
Materials and methods

\section{Study design and sample}

The Strengthening Human Resources for Health (HRH) Project (2012-2017) conducted a nationwide survey of job satisfaction, motivation, and turnover intentions among Ethiopian health workers at public-sector hospitals in 2014. A cross-sectional study design was used to gather nationally representative information on anesthetists at a randomly selected sample of 108 hospitals (out of the 122 total). Data collectors invited all eligible anesthetists at these hospitals to participate in the study. Anesthetists were eligible if they were full-time permanent hospital employees, had at least 6 months of work experience in anesthesia, and were available and willing to participate. Their training varied. Diploma Level 5 anesthetists are experienced nurses who have completed a 1-year Regional Health Science College training program. In the past, Advanced Diplomas were awarded to nurses with 2 years of anesthesia training, but this training track was discontinued. To receive a BSc in anesthesia, high school graduates must complete a 4-year university program, while experienced nurses must complete a 3-year university program. An MSc requires an additional 2-year university training program.

An adjusted minimum sample size of 232 anesthetists was calculated using statistical parameters of $95 \%$ confidence interval, expected proportion of intention to leave as 0.5 , a design effect of 1.2 , and a relative precision of $10 \%$. On average, 2.4 anesthetists (range 1-10) were interviewed at each of 104 hospitals in 10 of Ethiopia's 11 administrative regions and cities, for a total sample of 251 anesthetists; no anesthetists were available for interview at four hospitals.

\section{Data collection}

Data were collected from May 28 to June 14, 2014. Individual interviews were conducted in private in the national language, Amharic. Interviewers read a series of closedended questions aloud to each respondent from a structured questionnaire and recorded their answers. The questionnaire was adapted from a job satisfaction and motivation tool developed by the Capacity Project for use with health workers in Uganda [17]. No systematic studies exist on the reliability and validity of the tool. However, health workers' understanding of the questions was checked during a pretest of the tool in the Oromia Region, after which the wording and sequence of certain questions were revised to ensure their clarity and relevance to the Ethiopian setting. 
Interviewers asked respondents about their demographic characteristics and work history, intentions to leave the job, and perceptions of conditions at home and at work that might affect retention. To assess turnover intentions, interviewers asked a yes-or-no question: "Are you planning to leave your job in the next one year?" To assess perceptions of 20 items that studies suggest may affect health workers' decisions to leave their jobs, interviewers asked: "If you were planning to leave your job, how important would each of the following items be in that decision?" Respondents rated each item on a Likert scale, from 1 (not important) to 5 (extremely important).

All 24 interviewers had university degrees in health or social science, and one supervisor was assigned to each region. Supervisors were drawn from HRH Project staff. Data collectors and supervisors attended 2 days of training, which included role plays and practice interviews and covered consent procedures, research ethics, and interview techniques.

\section{Data analysis}

Data were entered into Epi-Info and exported to STATA 13.1 for cleaning and analysis. Exploratory factor analysis and a literature review [18] were used to group 20 decisionmaking items into six uncorrelated factors: living conditions, conditions at the workplace, relationship with supervisor and co-workers, work burden, opportunities for professional development, and basic salary. To check reliability and internal consistency of items in each factor, we calculated Cronbach's alpha coefficient [19]. Values exceeded the 0.7 cutoff for all factors except conditions at the workplace ( $\alpha=0.619)$. Chi-square test was used to test for associations between turnover intentions and demographic and work-related characteristics, with a $p$ value $<0.05$ used to determine statistical significance.

Bivariate analysis assessed the independent effect of each predictor on turnover intentions. Then a multivariable logistic regression model was fitted, using a stepwise (backward) variable selection process to extract potential predictors of turnover intentions. After entering all variables into the model, stepwise selection was used to remove those where $p>0.3$, leaving six variables. Multicollinearity among predictors was assessed to ensure reliability of the multivariable model. Adjusted odds ratios and 95\% confidence intervals were calculated to show the magnitude of associations.

\section{Ethical considerations}

The John Hopkins University Institutional Review Board approved the study protocol, and the Ethiopian FMOH gave permission to conduct the study. Before each interview, data collectors read a consent script in Amharic to obtain informed verbal consent. To ensure confidentiality, individual identifiers were not used during data collection and analysis.

\section{Results}

\section{Respondents' characteristics and turnover intentions}

Most respondents had a bachelor's degree ( $n=145$; $57.8 \%$ ) (Table 1). They had served in the public health system from 6 months to 37 years (median $=4.8$ years); for some, this included prior service as nurses. Almost twothirds ( $n=162 ; 64.5 \%$ ) were currently fulfilling a compulsory service obligation, with $2-72$ months remaining (data not shown).

Almost half ( $n=120 ; 47.8 \%$ ) planned to leave the job in the next year, but turnover intentions were significantly lower among those over age $30(n=36 ; 37.1 \%)$ and those with dependents $(n=83 ; 43.5 \%)$ (Table 1$)$. Turnover intentions exceeded $60 \%$ among anesthetists with 2-5 years of service and those working at district hospitals. Fulfillment of the compulsory service obligation did not predict intention to leave.

\section{Factors affecting decisions to leave the job}

Among the six decision-making factors, respondents rated basic salary (mean score $=4.52$; $\mathrm{SD}=1.0$ ) and professional development opportunities (mean score $=4.22$; $\mathrm{SD}=0.89$ ) as most important and living conditions as least important (mean score $=3.42$, SD $=0.89$ ). Conditions at the workplace (mean score $=3.72 ; \mathrm{SD}=0.94$ ), work burden (mean score $=3.54$; $\mathrm{SD}=1.2$ ), and relationship with supervisor and co-workers (mean score $=3.51 ; \mathrm{SD}=0.98$ ) were of intermediate importance. Table 2 shows how respondents rated individual items. Over $85 \%$ said low pay, limited opportunities for promotion, poor access to higher education, safety concerns, and high cost of living were important.

The bivariate analysis found that turnover intentions were associated with two decision-making factors (living conditions and professional development opportunities) plus age, dependents, years of service, and facility type (Table 3). The final multivariable model included six variables, five of which were significant predictors of turnover intentions. Intention to leave the job fell by $5 \%$ with each additional year in age (adjusted OR $=0.95,95 \%$ CI 0.92, 0.99). Anesthetists at referral hospitals were half as likely to intend to leave the job as those at district hospitals (adjusted OR $=0.48,95 \%$ CI $0.23,0.98$ ). The 
Table 1 Distribution of respondents and percent who intend to leave their jobs in the next year, by socio-demographic and work-related characteristics

\begin{tabular}{|c|c|c|c|c|c|}
\hline \multirow[t]{2}{*}{ Characteristic } & \multicolumn{2}{|c|}{ All respondents } & \multicolumn{3}{|c|}{ Respondents who intend to leave their jobs in the next year } \\
\hline & Number & Percent distribution $(n=251)$ & Number & $\%$ of total & $p$ value* \\
\hline Total & 251 & 100 & 120 & 47.8 & na \\
\hline \multicolumn{6}{|l|}{ Gender } \\
\hline Male & 188 & 74.9 & 95 & 50.5 & \multirow[t]{2}{*}{0.136} \\
\hline Female & 63 & 25.1 & 25 & 39.7 & \\
\hline \multicolumn{6}{|l|}{ Age } \\
\hline$\leq 30$ years & 153 & 61.2 & 83 & 54.2 & \multirow[t]{2}{*}{0.008} \\
\hline Over 30 years & 97 & 38.8 & 36 & 37.1 & \\
\hline \multicolumn{6}{|l|}{ Marital status } \\
\hline Single & 135 & 53.8 & 70 & 51.8 & \multirow[t]{2}{*}{0.055} \\
\hline Ever married & 116 & 46.2 & 50 & 45.0 & \\
\hline \multicolumn{6}{|l|}{ Dependents } \\
\hline Yes & 191 & 76.1 & 83 & 43.5 & \multirow[t]{2}{*}{0.014} \\
\hline No & 60 & 23.9 & 37 & 61.7 & \\
\hline \multicolumn{6}{|l|}{ Educational qualification } \\
\hline Master's degree & 18 & 7.2 & 5 & 27.8 & \multirow[t]{4}{*}{0.104} \\
\hline Bachelor's degree & 145 & 57.8 & 71 & 49.0 & \\
\hline Advanced Diploma & 36 & 14.3 & 14 & 38.9 & \\
\hline Diploma Level 5 & 52 & 20.7 & 30 & 57.7 & \\
\hline \multicolumn{6}{|c|}{ Years of service in public health system } \\
\hline 6 months -2 years & 83 & 33.1 & 41 & 49.4 & \multirow[t]{3}{*}{0.010} \\
\hline$>2-5$ years & 53 & 21.1 & 34 & 64.2 & \\
\hline$>5$ years & 115 & 45.8 & 45 & 39.1 & \\
\hline \multicolumn{6}{|c|}{ Currently fulfilling compulsory service obligation ${ }^{a}$} \\
\hline Yes & 162 & 64.5 & 78 & 48.1 & \multirow[t]{2}{*}{0.575} \\
\hline No & 89 & 35.5 & 42 & 47.2 & \\
\hline \multicolumn{6}{|l|}{ Type of facility } \\
\hline District hospital & 65 & 25.9 & 40 & 61.5 & \multirow[t]{3}{*}{0.033} \\
\hline Regional/zonal hospital & 94 & 37.4 & 42 & 44.7 & \\
\hline Referral hospital & 92 & 36.6 & 38 & 41.3 & \\
\hline
\end{tabular}

* Chi-square test

${ }^{a}$ The government requires anesthetists who graduate from a public educational institution to work (compulsory service) for 1-4 years in the public sector

more importance anesthetists attached to living conditions, the more likely they were to intend to quit (adjusted $\mathrm{OR}=1.83,95 \% \mathrm{CI} 1.17,2.86)$; the same was true for opportunities for professional development (adjusted $\mathrm{OR}=1.91,95 \%$ CI 1.26, 2.90). This pattern was reversed for conditions at the workplace: Anesthetists who considered this factor more important were less likely to intend to leave the job (adjusted OR $=0.63,95 \%$ CI 0.4, 0.99).

\section{Discussion}

Almost half $(n=120 ; 47.8 \%)$ of anesthetists said they planned to leave the job in the next year. Comparable data from other LMICs are rare, but a study in Thailand found similarly high levels of turnover intentions among nurse anesthetists [20]. Yet evidence suggests that most health workers do not act on stated turnover intentions. For example, actual annual turnover among midwives in Senegal was just 9\%, even though $58.9 \%$ reported their intention to quit within a year [21]. In Ethiopia, 59.4\% of all health workers at hospitals and health centers in Jimma 
Table 2 Percentage of anesthetists who consider items important in decisions to leave the job, mean and median scores

\begin{tabular}{|c|c|c|c|c|}
\hline \multirow[t]{2}{*}{ Items } & \multirow[t]{2}{*}{$N$} & \multirow[t]{2}{*}{$\%$ who say factor is important ${ }^{\mathrm{a}}$} & \multicolumn{2}{|l|}{ Score } \\
\hline & & & Mean & Median \\
\hline \multicolumn{5}{|l|}{ Living conditions } \\
\hline High cost of living & 251 & 87.2 & 4.07 & 5 \\
\hline Lack of housing facilities & 246 & 82.9 & 3.82 & 4 \\
\hline Poor/lack of utilities (water, electricity) at home & 249 & 78.7 & 3.49 & 4 \\
\hline Poor educational facilities for children ${ }^{\mathrm{b}}$ & 163 & 77.9 & 3.58 & 4 \\
\hline Transportation problems & 243 & 70.0 & 3.23 & 3 \\
\hline Work is far from home & 218 & 58.3 & 2.93 & 3 \\
\hline Lack of access to telephones to stay in touch with family and friends & 244 & 52.9 & 2.77 & 3 \\
\hline \multicolumn{5}{|l|}{ Conditions at the workplace } \\
\hline Concerns about safety at work ${ }^{c}$ & 251 & 89.2 & 4.08 & 4 \\
\hline Poor access to supplies and equipment at work & 251 & 79.7 & 3.63 & 4 \\
\hline Poor/lack of utilities (water, electricity, Internet) at work & 251 & 74.9 & 3.45 & 4 \\
\hline \multicolumn{5}{|l|}{ Relationship with supervisor and co-workers } \\
\hline Lack of recognition for good work & 249 & 83.6 & 3.78 & 4 \\
\hline Unfair treatment by a supervisor & 249 & 77.6 & 3.70 & 4 \\
\hline Poor supervision and feedback & 249 & 74.3 & 3.29 & 3 \\
\hline Social conflicts in the workplace & 249 & 73.9 & 3.28 & 4 \\
\hline \multicolumn{5}{|l|}{ Work burden } \\
\hline Heavy workload & 249 & 79.5 & 3.55 & 4 \\
\hline Long hours of work & 251 & 78.5 & 3.54 & 4 \\
\hline \multicolumn{5}{|l|}{ Opportunities for professional development } \\
\hline Poor access to higher education & 250 & 93.6 & 4.44 & 5 \\
\hline Limited opportunities for promotion & 250 & 92.4 & 4.32 & 5 \\
\hline Limited opportunities for in-service training & 251 & 83.7 & 3.88 & 4 \\
\hline \multicolumn{5}{|l|}{ Basic salary } \\
\hline Low pay & 250 & 93.6 & 4.52 & 5 \\
\hline
\end{tabular}

Response scale was: not important (1), somewhat important (2), important (3), very important (4), and extremely important (5)

${ }^{a}$ Includes responses of: extremely important, very important, and important

${ }^{\mathrm{b}}$ Question was only asked of respondents with children

${ }^{\mathrm{c}}$ Safety concerns focused on aging and unreliable anesthesia machines, exposure to infection due to lack of supplies, and physical trauma from moving heavy gas cylinders

Zone intended to leave their jobs in the next year or two compared with actual turnover of $45.9 \%$ over 5 years [22]. A record review in East Wollega Zone found an annual attrition rate of $19.3 \%$ for health workers holding diplomas or first degrees [23]. A 2012 baseline study conducted for the HRH Project found a $4 \%$ attrition rate for anesthetists in Ethiopia [24].

Even if personal relationships, limited job opportunities, or other factors prevent public-sector anesthetists from quitting, high turnover intentions signal that they are dissatisfied with their jobs and may be less motivated to perform well [25-27]. Therefore, the situation in Ethiopia demands action regardless of actual turnover levels, which this study did not document. The multivariable analysis identified five predictors of turnover intentions which can help devise effective strategies to reduce dissatisfaction and promote retention.

Intentions to leave the job declined with increasing age, confirming two studies of nurses in Ethiopia [14, 28]. Researchers have consistently found an inverse relationship between age and turnover intentions among nurses [18], and a multi-country study found that actual turnover among health workers over age 50 was half that of those under age 30 [25]. Younger health workers have fewer family ties and may leave a job to further their education or career. Compulsory service programs are designed to increase retention among this cohort, but have a mixed track record $[29,30]$. In this study, the government service obligation 
Table 3 Bivariate and multivariable logistic regressions: intention to leave the job in the next year (outcome), by socio-demographic characteristics and importance of decision-making factors

\begin{tabular}{|c|c|c|c|c|}
\hline \multirow[t]{2}{*}{ Predictors } & \multicolumn{2}{|c|}{ Bivariate logistic regression } & \multicolumn{2}{|c|}{ Multivariable logistic regression $^{\mathrm{a}}$} \\
\hline & Crude OR & $95 \% \mathrm{CI}$ & Adjusted OR & $95 \% \mathrm{CI}$ \\
\hline \multicolumn{5}{|l|}{ Gender } \\
\hline Male (ref.) & - & & & \\
\hline Female & 0.644 & $0.36-1.15$ & & \\
\hline Age in years & 0.952 & $0.92-0.98$ & 0.952 & $0.92-0.99$ \\
\hline \multicolumn{5}{|l|}{ Marital status } \\
\hline \multicolumn{5}{|l|}{ Single (ref.) } \\
\hline Ever married & 0.703 & $0.43-1.16$ & & \\
\hline \multicolumn{5}{|l|}{ Dependents } \\
\hline \multicolumn{5}{|l|}{ No (ref.) } \\
\hline Yes & 0.478 & $0.26-0.87$ & & \\
\hline \multicolumn{5}{|l|}{ Educational qualification } \\
\hline \multicolumn{5}{|l|}{ Master's degree (ref.) } \\
\hline Bachelor's degree & 2.494 & $0.85-7.36$ & & \\
\hline Diploma (Advanced or Level 5) & 2.600 & $0.85-7.91$ & & \\
\hline Years of public health service & 0.960 & $0.93-0.99$ & & \\
\hline \multicolumn{5}{|l|}{ Currently fulfilling compulsory service obligation } \\
\hline \multicolumn{5}{|l|}{ No (ref.) } \\
\hline Yes & 1.039 & $0.62-1.74$ & & \\
\hline \multicolumn{5}{|l|}{ Type of facility } \\
\hline \multicolumn{5}{|l|}{ District hospital (ref.) } \\
\hline Regional/zonal hospital & 0.505 & $0.27-0.96$ & 0.525 & $0.26-1.05$ \\
\hline Referral hospital & 0.440 & $0.23-0.84$ & 0.476 & $0.23-0.98$ \\
\hline \multicolumn{5}{|l|}{ Importance of decision-making factors } \\
\hline Living conditions & 1.534 & $1.14-2.06$ & 1.830 & $1.17-2.86$ \\
\hline Conditions at workplace & 1.195 & $0.92-1.56$ & 0.627 & $0.40-0.99$ \\
\hline Relationship with supervisor and co-workers & 1.033 & $0.80-1.33$ & & \\
\hline Work burden & 0.896 & $0.73-1.10$ & 0.789 & $0.61-1.03$ \\
\hline Professional development opportunities & 1.752 & $1.27-2.42$ & 1.912 & $1.26-2.90$ \\
\hline Basic salary & 1.213 & $0.94-1.57$ & & \\
\hline
\end{tabular}

$O R$ odds ratio, $C I$ confidence interval

${ }^{a}$ All factors were entered into the multivariable model, and then stepwise selection was used to remove from the full model those with $p>0.3$

did not predict turnover intentions, suggesting that other interventions are needed to retain younger anesthetists.

Turnover intentions were about twice as high at district hospitals as at regional and referral hospitals, even after controlling for variables related to living and working conditions. Attracting and retaining health workers at rural facilities like these is especially challenging and often requires special measures, such as recruiting students from and training them in the rural areas where they will be deployed, offering incentives for rural postings, and improving living and working conditions at rural facilities [31-33]. HR managers in Ethiopia should consider these kinds of interventions to retain anesthetists at district hospitals.

Turnover intentions increased with perceived importance of living conditions, which is consistent with previous studies. Inadequate living conditions increased job dissatisfaction and migration in Bangladesh [31], and housing had the greatest impact on non-physician health professionals choosing jobs at public health facilities in Mozambique [32]. The findings suggest that strategies such as housing allowances and improved access to water and electricity may reduce turnover intentions. Currently in Ethiopia, incentives related to housing are primarily directed to physicians. 
Turnover intentions also increased with perceived importance of professional development opportunities. A study in Jimma Zone identified lack of ongoing skills development and training as leading reasons frustrating retention of hospital and health workers [22]. A survey of anesthetists at 41 hospitals in Ethiopia found they had very limited access to refresher training, continuing professional development, and support from a physician anesthesiologist [34]. Lack of coaching and continuing education are common concerns expressed by anesthesia providers in other LMICs as well [4, 35, 36]. The findings suggest that expanding opportunities for coaching, continuing education, and advanced degrees may promote anesthetists' retention.

Contrary to expectations, turnover intentions declined with perceived importance of conditions at the workplace. Studies have documented serious deficits in supplies, equipment, and infrastructure for anesthesia in Ethiopia $[11,34]$, and the global literature suggests these deficits dampen morale of anesthetists in many LMICs [9, 35]. It is possible that the lack of internal consistency in the items making up this factor (which included safety and utilities as well as supplies and equipment) affected the results. Further investigation is needed to interpret this finding.

Researchers have applied the same tools and analytical approach used in this study to examine predictors of turnover intentions among nurses in Ethiopia [14]. Like anesthetists, half of nurses $(n=213 ; 50.2 \%)$ intended to leave the job in the next year. Few studies have compared turnover intentions of different health worker cadres, but they, too, found no significant differences [25, 37]. However, these studies only compared the levels, not the determinants, of turnover intentions. Notably, Ethiopian anesthetists have only one significant predictor of intention to leave in common with Ethiopian nurses: limited opportunities for professional development. For nurses, the two other significant predictors in a multivariable analysis were holding a university degree and fewer years of service. The divergence between anesthetists' and nurses' findings highlights the importance of separately assessing health worker cadres and tailoring retention strategies to each cadre's different situation to ensure their effectiveness.

This study fills an important information gap, because anesthetists have been a largely overlooked and understudied cadre. The large sample provides nationally representative information for policy making, but has some limitations. It did not include the private sector or ask whether respondents intended to leave the facility, the public sector, the country, or the profession-which may be driven by different factors. A further round of data collection to determine whether anesthetists acted on their turnover intentions could have produced actionable information on individuals most at risk of quitting.

\section{Conclusions}

High turnover intentions signal that anesthetists in Ethiopia are dissatisfied with their jobs and may suffer from low morale and motivation even if they do not actually quit. Findings suggest that the compulsory service requirement only delays anesthetists' exit from the public health system and that salary is not a significant predictor of their decisions. Strategies that focus on improving living conditions for anesthetists and expanding opportunities for professional development are likely to prove more effective. Age and location also matter: Anesthetists are more likely to plan on quitting when they are young and if they are posted to district hospitals. HR managers should consider special measures for anesthetists in these vulnerable categories.

Acknowledgements This work was supported by the United States Agency for International Development (USAID) under the Cooperative Agreement AID-663-A-12-00008. The contents are the responsibility of Jhpiego and do not necessarily reflect the views of USAID or the United States Government. We gratefully acknowledge Dr. Tegbar Yigzaw, Dr. Shelemo Kachara, Dr. Ummuro Adano, Dr. Damtew Woldemariam, Dr. Wendemagegn Eniable, Dr. Young Mi Kim, and the Ethiopian Association of Anesthetists, who helped design or review the study tools, and Mark Emerson, who supported the data analysis. Eureka Services PVT conducted the data collection. We thank the study participants for their time and viewpoints, and the Ethiopian Federal Ministry of Health and the Regional Health Bureaus for their leadership and facilitation of this study.

\section{Compliance with ethical standards}

\section{Conflict of interest None.}

Open Access This article is distributed under the terms of the Creative Commons Attribution 4.0 International License (http://crea tivecommons.org/licenses/by/4.0/), which permits unrestricted use, distribution, and reproduction in any medium, provided you give appropriate credit to the original author(s) and the source, provide a link to the Creative Commons license, and indicate if changes were made.

\section{References}

1. Meara J, Leather A, Hagander L et al (2016) Global Surgery 2030: evidence and solutions for achieving health, welfare, and economic development. Int J Obstet Anesth 25:75-78. doi:10. 1016/j.ijoa.2015.09.006

2. LeBrun D, Chackungal S, Chao $T$ et al (2014) Prioritizing essential surgery and safe anesthesia for the Post-2015 Development Agenda: operative capacities of 78 district hospitals in 7 low- and middle-income countries. Surgery 155:365-373. doi:10. 1016/j.surg.2013.10.008 
3. Federspiel F, Mukhopadhyay S, Milsom P et al (2015) Global surgical and anaesthetic task shifting: a systematic literature review and survey. Lancet 385:S46. doi:10.1016/S01406736(15)60841-8

4. Anderson R, Ahn R, Nelson B et al (2014) Defining the anesthesia gap for reproductive health procedures in resource-limited settings. Int J Gynaecol Obs 127:229-233. doi:10.1016/j.ijgo. 2014.06.023

5. Hendel S, Coonan T, Thomas S, McQueen K (2015) The ratelimiting step: the provision of safe anesthesia in low-income countries. World J Surg 39:833-841. doi:10.1007/s00268-0142775-9

6. Bergström S, McPake B, Pereira C, Workforce Innovations to Expand the Capacity for Surgical Services et al (2015) In: Debas H, Donkor P, Gawande A et al (eds) Essential surgery: disease control priorities, vol 1, 3rd edn. The World Bank, Washington, pp 307-316

7. Mavalankar D, Sriram V (2009) Provision of anaesthesia services for emergency obstetric care through task shifting in South Asia. Reprod Health Matters 17:21-31. doi:10.1016/S09688080(09)33433-3

8. Chu K, Rosseel P, Gielis P, Ford N (2009) Surgical task shifting in sub-Saharan Africa. PLoS Med 6(5):e1000078. doi:10.1371/ journal.pmed.1000078

9. Dubowitz G, Detlefs S, McQueen KA (2010) Global anesthesia workforce crisis: a preliminary survey revealing shortages contributing to undesirable outcomes and unsafe practices. World $\mathrm{J}$ Surg 34:438-444. doi:10.1007/s00268-009-0229-6

10. Hoyler M, Finlayson SRG, McClain CD et al (2014) Shortage of doctors, shortage of data: a review of the global surgery, obstetrics, and anesthesia workforce literature. World J Surg 38:269-280. doi:10.1007/s00268-013-2324-y

11. Chao TE, Burdic M, Ganjawalla K et al (2012) Survey of surgery and anesthesia infrastructure in Ethiopia. World J Surg 36:2545-2553. doi:10.1007/s00268-012-1729-3

12. Federal Democratic Republic of Ethiopia Ministry of Health (2016) National Human Resource for Health Strategic Plan for Ethiopia 2016-2025. Ministry of Health, Addis Ababa

13. Feysia B, Herbst CH, Lemma W et al (2012) The Health Workforce in Ethiopia: addressing the remaining challenges. The World Bank, Washington. http://documents.worldbank.org/cura ted/en/433741468250204395/pdf/662180PUB0EPI00pia0health0 workforse.pdf

14. Ayalew F, Kols A, Kim YM et al (2015) Factors affecting turnover intention among nurses in Ethiopia. World Health Popul $16: 62-74$

15. Africa Health Workforce Observatory (2010) Human resources for health country profile: Ethiopia. African Health Workforce Observatory, Brazzaville. http://s3.amazonaws.com/zanran_sto rage/www.hrh-observatory.afro.who.int/ContentPages/24532 20519.pdf

16. Kaur B, Mohindru Pankaj (2013) Antecedents of turnover intentions: a literature review. Glob J Manag Bus Stud 3:1219-1230

17. Capacity Project (2006) Job Satisfaction Survey (Draft). Capacity Project, Chapel Hill. http://www.hrhresourcecenter.org/node/910

18. Hayes LJ, O'Brien-Pallas L, Duffield C et al (2012) Nurse turnover: a literature review-an update. Int J Nurs Stud 49:887-905. doi:10.1016/j.ijnurstu.2011.10.001

19. Pallant J (2007) SPSS survival manual: a step by step guide to data analysis using SPSS for Windows, 3rd edn. Open University Press, New York

20. Apidechakul P, Vichitvejpaisal P, Chainchop P et al (2015) Factors influencing intentions to leave of nurse anesthetists in a public university hospital. Thai J Anesthesiol 41:254-262
21. Rouleau D, Fournier P, Philibert A et al (2012) The effects of midwives' job satisfaction on burnout, intention to quit and turnover: a longitudinal study in Senegal. Hum Resour Health 10:9. doi:10.1186/1478-4491-10-9

22. Gesesew H, Tebeje B, Alemseged F et al (2016) Health workforce acquisition, retention and turnover in southwest Ethiopian health institutions. Ethiop J Heal Sci 26:331-340

23. Hailemichael Y, Jira C, Girma B et al (2010) Health workforce deployment, attrition and density in East Wollega zone, Western Ethiopia. Ethiop J Health Sci 20:15-23

24. Jhpiego Ethiopia (2013) Strengthening Human Resources for Health (HRH) Program: baseline survey findings. Jhpiego/ Ethiopia, Addis Ababa

25. Blaauw D, Ditlopo P, Maseko F et al (2013) Comparing the job satisfaction and intention to leave of different categories of health workers in Tanzania, Malawi, and South Africa. Glob Health Action 6:19287. doi:10.3402/gha.v6i0.19287

26. Bonenberger M, Aikins M, Akweongo P et al (2014) The effects of health worker motivation and job satisfaction on turnover intention in Ghana: a cross-sectional study. Hum Resour Health 12:43. doi:10.1186/1478-4491-12-43

27. Franco LM, Bennett S, Kanfer R (2002) Health sector reform and public sector health worker motivation: a conceptual framework. Soc Sci Med 54:1255-1266

28. Engeda EH, Birhanu AM, Alene KA (2014) Intent to stay in the nursing profession and associated factors among nurses working in Amhara Regional State Referral Hospitals, Ethiopia. BMC Nurs 13:24. doi:10.1186/1472-6955-13-24

29. Frehywot S, Mullan F, Payne PW et al (2010) Compulsory service programmes for recruiting health workers in remote and rural areas: do they work? Bull World Health Organ 88:364-370. doi:10.2471/BLT.09.071605

30. Liu X, Dou L, Zhang H et al (2015) Analysis of context factors in compulsory and incentive strategies for improving attraction and retention of health workers in rural and remote areas: a systematic review. Hum Resour Health 13:61. doi:10.1186/s12960-0150059-6

31. Darkwa EK, Newman MS, Kawkab M et al (2015) A qualitative study of factors influencing retention of doctors and nurses at rural healthcare facilities in Bangladesh. BMC Health Serv Res 15:344. doi:10.1186/s12913-015-1012-z

32. Honda A, Vio F (2015) Incentives for non-physician health professionals to work in the rural and remote areas of Mozambique-a discrete choice experiment for eliciting job preferences. Hum Resour Health 13:23. doi:10.1186/s12960-015-0015-5

33. Strasser R, Kam SM, Regalado SM (2016) Rural health care access and policy in developing countries. Annu Rev Public Health 37:395-412. doi:10.1146/annurev-publhealth-032315021507

34. Bashford T (2014) Anesthesia in Ethiopia: providers perspectives on the current state of the service. Trop Dr 44:6-13. doi:10.1177/ 0049475513512642

35. Collins SB (2011) Model for a reproducible curriculum infrastructure to provide international nurse anesthesia continuing education. AANA J 79:491-496

36. Ross O, Flutter L, Dali L et al (2012) Anaesthesia Assistant Follow-up and Enhancement Programme, Nepal 2011-2012. Nick Simons Institute, Kathmandu. http://www.aagbi.org/sites/ default/files/ROSS,\%20Oliver\%20-\%20AA\%20FEP\%20report. pdf

37. Fogarty L, Kim YM, Juon HS et al (2014) Job satisfaction and retention of health-care providers in Afghanistan and Malawi. Hum Resour Health 12:11. doi:10.1186/1478-4491-12-11 